\title{
The Incidence of Cerebrovascular Lesions in Vascular Dementia Due to Arteriosclerosis and Due to Cerebral Amyloid Angiopathy: A Post-Mortem Neuropathological Study With 7.0-Tesla Magnetic Resonance Imaging
}

\section{Jacques De Reuck*, Florent Auger, Nicolas Durieux, Claude-Alain Maurage, Vincent Deramecourt, Charlotte Cordonnier, Florence Pasquier, Didier Leys and Regis Bordet}

Unitè 1171 "Degenerative and vascular cognitive disorders", Université de Lille 2, Lille, France

*Corresponding Author: Jacques De Reuck, Unitè 1171 "Degenerative and vascular cognitive disorders", Université de Lille 2, Lille, France.
Received: January 25, 2020

Published: February 29, 2020

(C) All rights are reserved by Jacques De

Reuck, et al.

\begin{abstract}
Introduction and Purpose: Arteriosclerotic small vessel disease (Art) is the main cause of vascular dementia (VaD). Cerebral amyloid angiopathy (CAA) can also lead to VaD. However it is not known whether the responsible cerebrovascular lesions and their severity are the same in VaD-Art and VaD-CAA. The present post-mortem study with additional 7-tesla magnetic resonance imaging (MRI) compares the incidence and the distribution of these lesions in both VaD disease types.

Material and Methods: 32 brains with pure VaD due to Art and due to CAA, and 31 with VaD-Art and VaD-CAA mixed with additional neurodegenerative pathology were compared separately. MRi was mainly used to compare the regional severity of the white matter changes (WMCs) and the incidence of cortical micro-infarcts (CoMIs) and cortical micro-bleeds (CoMBs) on serial cerebral hemispheric sections between both groups of VaD.

Results: VaD-CAA was characterized by the more frequent occurrence of CoMIs and lobar haematomas while in VaD-Art lacunar infarcts predominated in the pure as well in the mixed forms. On MRI WMCs were more severe in the central section and to a minor degree in the mixed VaD-CAA, as well as CoMiBs in the frontal section. CoMIs were equally increased in the different sections.

Conclusions: There are significant differences in the severity and the topographic distributions of cerebrovascular lesions between VaD-Art and VaD-CAA brains. However, the increased WMCs and CoMiBs in the mixed form of VaD-CAA could possibly be related to the additional neurodegenerative changes.
\end{abstract}

Keywords: Neuropathology; Post-Mortem Magnetic Resonance Imaging; Cerebrovascular Lesions; Arteriosclerotic Vascular Dementia; Vascular Dementia Due to Cerebral Amyloid Angiopathy

\section{Introduction}

Cerebrovascular lesions are a frequent finding in the elderly population. However, the impact of these lesions on cognitive performance, the prevalence of vascular dementia (VaD), and the pathophysiology behind the characteristics In vivo imaging findings are subject to controversy [1]. There are no generally accepted protocols for post-mortem assessment in cases of suspected vascular cognitive impairment [2]. The most described morphological types are multi-infarct encephalopathy, strategic infarcts, subcortical arteriosclerotic leukoencephalopathy, multi-lacunar state and post-ischaemic encephalopathy [3]. However, in recent years cerebral small vessel disease (SVD) has gained prominence worldwide 
The Incidence of Cerebrovascular Lesions in Vascular Dementia Due to Arteriosclerosis and Due to Cerebral Amyloid Angiopathy: A Post-Mortem Neuropathological Study With 7.0-Tesla Magnetic Resonance Imaging

as the most important substrate of vascular cognitive impairment. Arteriosclerosis (Art) of SVD, lacunar infarcts (LIs), cortical micro-infarcts (CoMIs) and diffuse white matter changes (WMCs), involving myelin loss and axonal abnormalities are considered to be the main contributively lesions [4].

However, also severe cerebral amyloid angiopathy (CAA) is also an underestimated cause of $\mathrm{VaD}$ [5]. The latter is biochemically closely related to Alzheimer dementia (AD) [6]. CAA appears to be in part a protein elimination failure angiopathy, characterized by the occurrence of lobar haematomas (LHs), cortical micro-bleeds (CoMBs), CoMIs, cortical superficial siderosis and WMCs [7].

In elderly brains mixed dementia predominates, mainly due to the association of Lewy body disease (LBD) and cerebrovascular pathologies to $\mathrm{AD}$ [8]. CAA related lesions are also more common in the mixed cases of AD, LBD and VaD [9].

In the pure and mixed cases of VaD no studies have been performed to compare the frequency and the topographic distribution of the different types of cerebrovasular lesions related to Art and to CAA.

The present post-mortem study compares the incidence of these lesions with additional 7.0-tesla magnetic resonance imaging of the distribution of CoMIs, CoMBs and WMCs on serial brain sections.

\section{Material and Methods}

Sixty-three patients with cognitive decline due to VaD, who had been followed up at the Lille University Hospital, underwent an autopsy. A previously obtained informed consent from the nearest family allowed an autopsy for diagnostic and scientific purposes. The brain tissue samples were acquired from the Lille Neuro-Bank of the Lille University, federated to the "Centre des Resources Biologiques" that acted as an institutional review board.

In addition to the macroscopic assessment of the cerebrovascular lesions the standard diagnostic procedure for the evaluation of eventual additional neurodegenerative and small vascular pathology included histological samples from the primary motor cortex, the associated frontal, temporal and parietal cortex, the primary and secondary visual cortex, the cingulate gyrus, the basal nucleus of Meynert, the amygdaloid body, the hippocampus, the basal ganglia, the mesencephalon, the pons, the medulla and the cerebellum.
Slides from paraffin embedded sections were stained with haematoxylin-eosin, luxol fast blue and Perl. Immune-staining for protein tau, $\beta$-amyloid, $\alpha$-synuclein, prion protein, TDP-43 and ubiquitin was also performed.

The diagnosis of CAA was retained when a significant score of 3 was reached in at least 4 cortical samples according to a recent consensus protocol [10].

In addition to the diagnostic procedure a whole coronal section of a cerebral hemisphere at the level of the mamillary body was used to evaluate to severity of the WMCs and the incidence of CoMIs and CoMBs.

On post-mortem examination 31 brains had a pure form of $\mathrm{VaD}$, while in 32 brains they were mixed with neurodegenerative pathology. Fourteen brains of the former group were related to Art, while 17 were due to severe CAA. In the mixed groups Art was the cause in $11 \mathrm{VaD}$ brains and in 21 mixed cases with pronounced CAA.

In the VaD- Art group the additional neurodegenerative pathology was in 9 cases AD grade 2 - 4 [11], in one case LBD and in another frontotemporal lobar degeneration. In the VaD-CAA 19 cases of AD grade 2 - 4 and 2 cases with additional LBD pathology were observed.

A 7.0-tesla MRI Bruker BioSpin SA was used with an issuer-receiver cylinder coil of $72 \mathrm{~mm}$ inner diameter (Ettlingen, Germany), according to a previously described method [12]. Three coronal sections of a cerebral hemisphere were submitted to T2 and T2* MRI sequences: a frontal one, a central one and one at the level of the parietal lobe. Semi-quantitative assessment of the severity of the WMCs and the frequency of the CoMIs and the CoMBs was determined in each coronal section separately.

Univariate comparisons of unpaired groups were performed with the Fisher's exact test for categorical data. The non-parametric Mann-Whitney U-test was used to compare continuous variables. The significance level, two-tailed, was set at $\leq 0.001$ for highly significant, $\leq 0.01$ for significant and $\leq 0.05$ for marginally significant.

\section{Results}

The average age of the patients with pure VaD-Art and VaD-CAA was similar with respectively 75 (SD: 9) and 76 (SD: 11) years. 
The neuropatholgical evaluation showed no differences of the severity of WMCs and, of the incidence of territorial infarcts and CoMBs between both groups. On the other hand LIs were more frequent in the VaD-Art brains while lobar haematomas (LHs) and CoMIs predominate in the VaD-CAA group (Table 1). On MRI examination only CoMIs were significantly more frequent in all the sections of the VaD-CAA brains (Table 2 and Figure 1).

\begin{tabular}{|l|c|c|c|}
\hline Items & VaD-Art & VaD-CAA & P Value \\
\hline White matter changes & $1.4(0.6)$ & $1.7(0.6)$ & N.S \\
\hline Lacunar infarcts & $1.4(0.9)$ & $0.6(0.7)$ & $<0.001$ \\
\hline Territorial infarcts & $0.8(0.6)$ & $0.6(0.7)$ & N.S \\
\hline Lobar haematomas & $0.3(0.5)$ & $0.7(0.6)$ & $<0.05$ \\
\hline Cortical micro-infarcts & $1.3(0.9)$ & $3.3(1.5)$ & $<0.001$ \\
\hline Cortical micro-bleeds & $1.6(0.6)$ & $2.2(1.0)$ & N.S \\
\hline
\end{tabular}

Table 1: Comparison of frequency of cerebrovascular lesions (standard deviation) in pure vascular dementia due to arteriosclerotic small vessel disease (VaD-Art) and cerebral amyloid angiopathy (VaD-CAA).

\begin{tabular}{|l|c|c|c|}
\hline Items & Vad-Art & Vad-Caa & P Value \\
\hline White matter changes & & & \\
\hline Frontal section & $0.8(0.9)$ & $1.0(0.9)$ & N.S \\
\hline Central section & $0.8(0.7)$ & $1.3(0.9)$ & N.S \\
\hline Posterior section & $0.8(0.8)$ & $1.2(0.8)$ & N.S \\
\hline Cortical micro-infarcts & & & \\
\hline Frontal section & $1.3(0.8)$ & $2.1(0.9)$ & $<0.01$ \\
\hline Central section & $0.7(0.6)$ & $2.1(0.9)$ & $<0.001$ \\
\hline Posterior section & $0.5(0.8)$ & $2.4(0.9)$ & $<0.001$ \\
\hline Cortical micro-bleeds & & & \\
\hline Frontal section & $2.5(0.5))$ & $2.0(1.0)$ & N.S \\
\hline Central section & $2.2(0.7)$ & $2.0(0.9)$ & N.S \\
\hline Posterior section & $2.6(0.5)$ & $2.0(1.0)$ & N.S \\
\hline
\end{tabular}

Table 2: Comparison of the distribution of the white matter changes, cortical micro-infarcts and cortical micro-bleeds (standard deviation) on 7.0-tesla magnetic resonance imaging in pure vascular dementia due to arteriosclerotic small vessel disease (VaD-Art) and cerebral amyloid angiopathy (VaD-CAA).

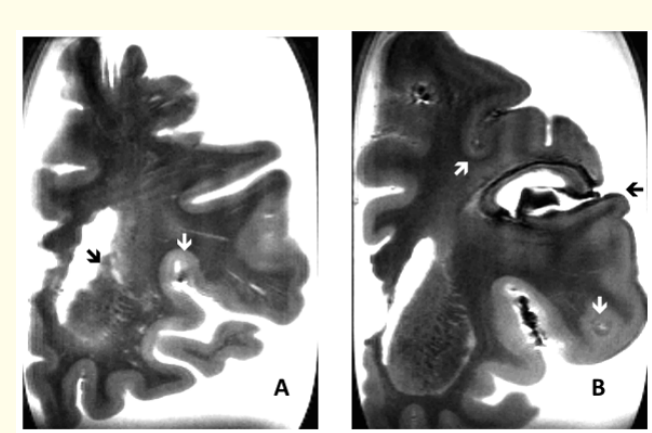

Figure 1: MRI T2 sequence of frontal sections of brains with pure vascular dementia. A: a lacunar infarct (black arrow) and a cortical micro-infarct (white arrows) in the arteriosclerotic type. B: old lobar haematoma (black arrow) and several cortical micro-infarcts (white arrows) in the cerebral amyloid angiopathy type.

The average age of the patients with mixed VaD-Art and VaDCAA was somewhat older than those with pure VaD, without a significant difference between both groups. The average age was respectively of 79 (SD: 9) and 77 (SD: 11) years.

The neuropathological examination revealed that the severity of the WMCs and the incidence of territorial infarcts were similar between both groups. The predominance of LIs in the VaD-Art brains and, of the CoMIs and LHs in the VaD-CAA ones remained as observed in the pure forms (Table 3).

\begin{tabular}{|l|c|c|c|}
\hline Items & $\begin{array}{c}\text { Mixed } \\
\text { Vad-Art }\end{array}$ & $\begin{array}{c}\text { Mixed } \\
\text { Vad-Caa }\end{array}$ & P Value \\
\hline White matter changes & $1.5(0.7)$ & $1.8(0.6)$ & N.S \\
\hline Lacunar infarcts & $1.8(0.8)$ & $1.1(0.7)$ & $<0.05$ \\
\hline Territorial infarcts & $1.0(0.7)$ & $0.9(0.8)$ & N.S \\
\hline Lobar haematomas & $0.3(0.4)$ & $0.6(0.3)$ & $<0.05$ \\
\hline Cortical micro-infarcts & $1.6(0.6)$ & $2.1(0.5)$ & $<0.05$ \\
\hline Cortical micro-bleeds & $1.7(0.6)$ & $1.8(0.8)$ & N.S \\
\hline
\end{tabular}

Table 3: Comparison of frequency of cerebrovascular lesions (standard deviation) in mixed vascular dementia due to arteriosclerotic small vessel disease (VaD-Art) and cerebral amyloid amgiopathy (VaD-CAA). 
The Incidence of Cerebrovascular Lesions in Vascular Dementia Due to Arteriosclerosis and Due to Cerebral Amyloid Angiopathy: A Post-Mortem Neuropathological Study With 7.0-Tesla Magnetic Resonance Imaging

On MRI examination WMCs were most severe in central and to some lesser degree in the frontal section of VaD-CAA brains compared to those with VaD-Art. CoMIs were more common in all the sections of VaD-CAA brains (Figure 2). CoMBs were only moderately more frequent in the frontal region of this group (Table 4) (Figure 3).

A

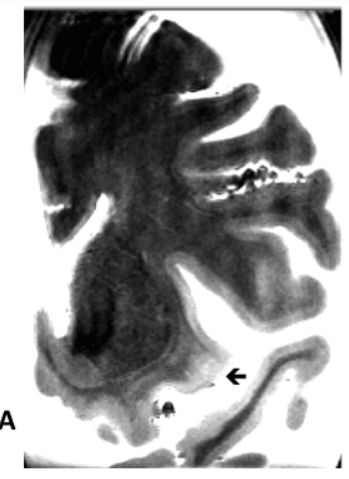

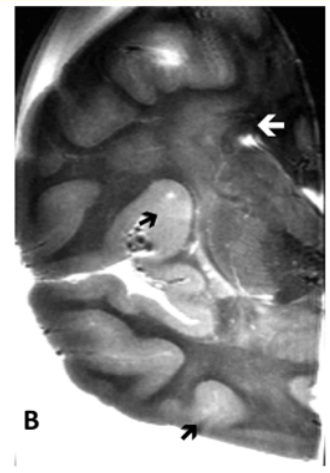

Figure 2: MRI T2 sequence of frontal sections of brains with mixed vascular dementia. A: cortical micro-infarct (black arrow) in the arteriosclerotic type. B: cortical micro-infarcts (black arrow) and confluent white matter changes (white arrow) in the cerebral amyloid angiopathy type.
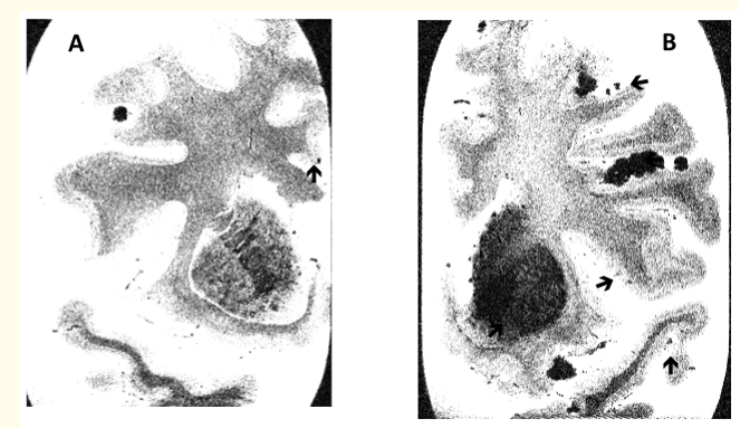

Figure 2: MRI T2* sequence of frontal sections of brains with mixed vascular dementia. A: cortical micro-bleed (black arrows) in the arteriosclerotic type. B: several cortical micro-beeds (black arrow) in the cerebral amyloid angiopathy type.

\begin{tabular}{|l|c|c|c|}
\hline Items & $\begin{array}{c}\text { Mixed } \\
\text { VaD-Art }\end{array}$ & $\begin{array}{c}\text { Mixed } \\
\text { VaD-CAA }\end{array}$ & P Value \\
\hline White matter changes & & & \\
\hline Frontal section & $0.8(0.6)$ & $1.3(0.5)$ & $<0.05$ \\
\hline Central section & $0.8(0.7)$ & $1.9(0.6)$ & $<0.001$ \\
\hline Posterior section & $0.8(0.8)$ & $0.9(0.8)$ & N.S \\
\hline Cortical micro-infarcts & & & \\
\hline Frontal section & $1.3(0.8)$ & $2.6(0.7)$ & $<0.001$ \\
\hline Central section & $0.7(0.6)$ & $2.0(0.7)$ & $<0.001$ \\
\hline Posterior section & $2.5(0.8)$ & $3.1(0.4)$ & $<0.05$ \\
\hline Cortical micro-bleeds & & & \\
\hline Frontal section & $2.5(0.5))$ & $3.1(1.4)$ & $<0.05$ \\
\hline Central section & $2.2(0.7)$ & $2.5(0.5)$ & N.S \\
\hline Posterior section & $3.0(0.7)$ & $3.4(0.5)$ & N.S \\
\hline
\end{tabular}

Table 4: Comparison of the distribution of the white matter changes, cortical micro-infarcts and cortical micro-bleeds (standard deviation) on 7.0-tesla magnetic resonance imaging in mixed vascular dementia due to arteriosclerotic small vessel disease (VaD-Art) and cerebral amyloid angiopathy (VaD-CAA).

\section{Discussion}

The present study confirms that in the pure as well as in mixed forms of VaD-CAA CoMIs and LHs are more frequent than in VaD-Art, while LIs are more frequent in the latter. Also in the mixed VaD-CAA there is some increase of WMCs in the frontal and central sections and of CoMBs in the frontal section on MRI examination.

A weak point of the study is the somewhat restricted number of patients in the different subgroups. Also, we cannot exclude that the mixed group of VaD-CAA had some minor degree of Art.

Although our patients with mixed dementia are somewhat older than those with pure VaD, still the latter can also occur in old age [13].

Medicated hypertension and ischaemic heart disease are risk factors associated to the occurrence of SVD [14]. They are, however, as a whole significantly associated with VaD-Art as well as with VaD-CAA at death [15].

In one study CAA and CoMIs are considered as the most putative substrates in VaD [16]. In another post-mortem study CAA is also 
The Incidence of Cerebrovascular Lesions in Vascular Dementia Due to Arteriosclerosis and Due to Cerebral Amyloid Angiopathy: A Post-Mortem Neuropathological Study With 7.0-Tesla Magnetic Resonance Imaging

related to the increase of CoMIs but not considered as the most important factor in the vascular pathology [17]. Our study shows, on the other hand, that in VaD associated to mild AD features CAA is a significant contributor for increase of cerebrovascular lesions [18]. Also these lesions are significantly more frequent in pure CAA cases without $\mathrm{AD}$ compared to those with severe AD disease [19].

Although CAA in pure and in mixed VaD is responsible for the most severe cerebrovascular lesions, the question still remains whether the increase of severity of WMCs in the mixed form is the consequence of CAA or due to the associated neurodegenerative pathology [20]. ApoE genotype is a major risk factor not only for neurodegenerative dementia but also for CAA [21]. Frontal lobe WMCs are related to neurofibrillary pathology in the aging brain [22].

CoMBs are also more related to the neurodegenerative process than to presence of CAA and reflect associated disruption of the blood-brain barrier [23].

So, the increased WMCs in the frontal and central sections and the CoMBs in the frontal lobe on MRI in the mixed VaD-CAA are most probably secondary to the associated neurodegenerative changes in the cerebral cortex rather than due to the CAA itself.

In conclusion CAA is responsible for the most severe cerebrovascular lesions in VaD with a further extension in the mixed form.

\section{Disclosure}

The authors have nothing to declare in relation to this article. No funding was received for the publication of this article.

\section{Bibliography}

1. McAleese KE., et al. "Post-mortem assessment in vascular dementia: advances and aspirations". BMC Medicine 14. 01 (2016): 129.

2. Skrobot OA., et al. "Vascular cognitive impairment neuropathology guidelines (VCINFG): the contribution of cerebrovascular pathology to cognitive impairment". Brain 139.11 (2016): 2957-2969.

3. Jellinger KA. "Pathogenesis and treatment of vascular cognitive impairment". Neurodegenerative Disease Management 04.06 (2014): 471-490.

4. Kalaria RN. "The pathology and pathophysiology of vascular dementia". Acta Neuropathologica 131.05 (2016): 659-685.
5. Haglund M., et al. "Severe cerebral amyloid angiopathy characterizes an underestimated variant of vascular dementia". Dementia and Geriatric Cognitive Disorders 18.02 (2004): 132137.

6. Vinters HV., et al. "Review: Vascular dementia: clinicopathologic and genetic considerations". Neuropathology and Applied Neurobiology 44.03 (2018): 247-266.

7. Charidimou A., et al. "Emerging concepts in sporadic cerebral amyloid angiopathy”. Brain140.07 (2017): 1829-1850.

8. De Reuck J., et al. "Cerebrovasular lesions in mixed neurodegenerative dementia: A neuropathological and magnetic resonance study". European Neurology 78.1-2 (2017): 1-5.

9. De Reuck J., et al. "Aging and cerebrovascular lesions in pure and mixed neurodegenerative and vascular dementia brains: a neuropathological study". Folia Neuropathologica 56.02 (2018): 81-87.

10. Love S., et al. "Development, appraisal, validation and implementation of a consensus protocol for the assessment of cerebral amyloid angiopathy in post-mortem brain tissue". American Journal of Neurodegenerative Diseases 03.01 (2014): 19-32.

11. Braak H., et al. "Staging of Alzheimer-related cortical destruction". European Neurology 33.06 (1993): 403-408.

12. De Reuck J., et al. "Comparison of 7.0-T T2* magnetic resonance imaging of cerebral bleeds in post-mortem brain sections of Alzheimer patients with their neuropathological correlates". Cerebrovascular Disease 31.05 (2011): 511-517.

13. Jellinger KA and Attems J. "Is there pure vascular dementia in old age?" Journal of Neurological Sciences 299.1-2 (2010): $150-154$

14. Richardson K., et al. "The neuropathology of vascular disease in the Medical Research Council Cognitive Function and Ageing Study (MRC CFAS)". Current Alzheimer Research 09.06 (2012): 687-696.

15. Ince PG., et al. "Medical research council cognitive function and ageing neuropathology study". Neuropathology and Applied Neurobiology 43.05 (2017): 409-418.

16. Haglund M., et al. "Cerebral amyloid angiopathy and cortical microinfarcts as putative substrates of vascular dementia". International Journal of Geriatric Psychiatry 21.07 (2006): 681687. 
The Incidence of Cerebrovascular Lesions in Vascular Dementia Due to Arteriosclerosis and Due to Cerebral Amyloid Angiopathy: A Post-Mortem Neuropathological Study With 7.0-Tesla Magnetic Resonance Imaging

17. Kovari E., et al. "The relation between cerebral amyloid angiopathy and cortical microinfarcts in brain ageing and $\mathrm{Al}$ zheimer's disease". Neuropathology and Applied Neurobiology 39.05 (2013): 498-509.

18. DeReuck J., et al. "Impact of mild Alzheimer changes in vascular dementia: A neuropathological study". Acta Scientific Neurology 02.06 (2019): 37-42.

19. De Reuck J., et al. "Cerebrovascular lesions in cerebral amyloid angiopathy with and without Alzheimer's disease: A neuropathological study with post-mortem 7.0-tesla magnetic resonance imaging". EC Neurology 10.11 (2018): 954-960.

20. Leys D., et al. "Could Wallerian degeneration contribute to "leukoaraiosis" in subjects free of any vascular disorder?" Journal of Neurology, Neurosurgery and Psychiatry 54.01 (1991): 46-50.

21. Eseri M., et al. "Cerebral amyloid angiopathy, subcortical white matter disease and dementia: literature review and study in OPTIMA". Brain Pathology 25.01 (2015): 51-62.

22. Polvikoski TM., et al. "Frontal lobe white matter hyperintensities and neurofibrillary pathology in the oldest old". Neurology 75.23 (2010): 2071-2078.

23. De Reuck J. "The significance of small cerebral bleeds in neurodegenerative dementia syndromes". Aging Disease 03.04 (2012): 307-312.

\section{Assets from publication with us}

- Prompt Acknowledgement after receiving the article

- Thorough Double blinded peer review

- Rapid Publication

- Issue of Publication Certificate

- High visibility of your Published work

Website: https://www.actascientific.com/

Submit Article: https://www.actascientific.com/submission.php Email us: editor@actascientific.com

Contact us: +919182824667

Citation: Jacques De Reuck., et al. "The Incidence of Cerebrovascular Lesions in Vascular Dementia Due to Arteriosclerosis and Due to Cerebral Amyloid Angiopathy: A Post-Mortem Neuropathological Study With 7.0-Tesla Magnetic Resonance Imaging". Acta Scientific Neurology 3.3 (2020): 27-32. 